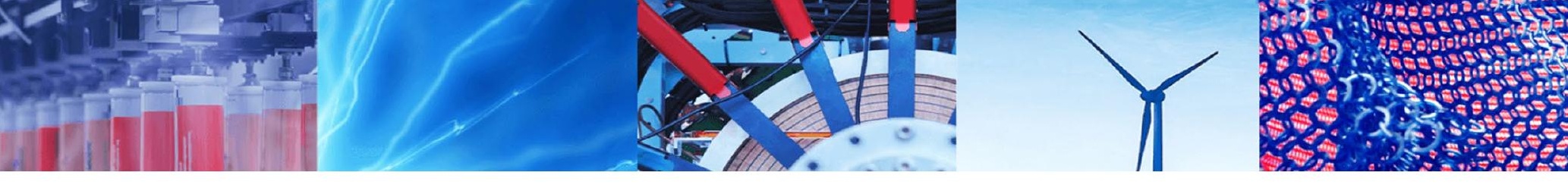

Research Article

\title{
Stokes flow and heat transfer past a circular cylinder in a square cavity with suction/injection on opposite-side walls
}

\author{
Pavankumar Reddy Muduganti ${ }^{1}$ [ $\cdot$ Ramana Murthy Venkata Josyula ${ }^{1}$
}

Received: 17 October 2019 / Accepted: 12 February 2020 / Published online: 27 February 2020

(c) Springer Nature Switzerland AG 2020

\begin{abstract}
In this paper, we study laminar viscous fluid flow about a circular cylinder placed in a square cavity of uniform crosssection generated by applying injection/suction at the opposite-side walls. Constant temperature is maintained on the walls without suction and on the boundary of the cylinder and constant heat flux is maintained on the walls with suction. In the literature, the problems dealing with flow past a cylinder in a cavity are very a few. The fluid flow pattern in the form of stream lines and temperature distribution in the form of isothermal lines are studied. The flow is assumed to be Stokesian and hence the resulting coupled equations for stream function and vorticity, and the energy equation for temperature are solved numerically by using the standard 5-point formula. Fictitious nodes are introduced for derivative boundary conditions for stream function by using central-difference scheme and 3-point backward difference formula is used for derivative boundary conditions on temperature. It is observed that the temperature increases drastically when a cylinder is kept in the cavity, i.e. it makes the quick heat transfer. Similarly, when a cylinder is placed near to a corner, the heat transfer is more rapid. Suction enhances the heat transfer.
\end{abstract}

Keywords Bi-harmonic equation · Stream lines · Vorticity contours · Isothermal lines

\section{Introduction}

The motion of a fluid past a cylindrical obstruction with its longitudinal axis aligned normal to the approaching flow has practical importance and is of fundamental interest. Bluff body cross-flow configurations arise in several industrial applications and environmental settings, which includes: the passages in equipment used for heat- and mass transfer processes; the cooling of electronic components and equipment; flow-metering devices; moving ground vehicles; the obstructed spaces between co-rotating disks in magnetic disk storage devices; tall buildings and structures such as cooling towers, chimneys, offshore oil rigs and electrical pylons. Hence, the heat transfer and flow characteristics of the flow past a stationary-isolated circular cylinder were well studied by researchers.
Williamson [1], Henderson [2, 3], Norberg [4], Baranyi and Lewis [5].

The flow field affected by suction/injection across the walls is encountered in fuel cell manifolds, micro-reactor channels, filtration units and in membrane reactor ducts. These problems of heat transfer and fluid flow were studied by many researchers by numerical methods in different situations such as (i) a square obstruction is placed asymmetrically between two sliding walls [6], (ii) natural convection between a hot horizontal cylinder placed in a square cavity [7], (iii) a horizontal cylinder is placed inside a rectangular enclosure [8], (iv) flow about a square cylinder [9], by using two numerical methods: FVM and lattice Boltzmann method. (v) the effects of grid resolution on simulations of large-eddy due to the flow about a circular pipe [10], (vi) the heat transfer in between the concentric

Pavankumar Reddy Muduganti, mprnitw@gmail.com | 'Department of Mathematics, National Institute of Technology, Warangal, Telangana 506004, India. 
and eccentric horizontal cylinders [11], (vii) natural convection is generated due to the temperature difference between a hot circular cylinder placed inside a cold square cavity [12], (viii) unsteady flow problem of power law fluid about a lengthy square tube [13], (ix) natural convection when the surface of circular cylinder is subjected to an uniform heat source within a square enclosure filled with air [14], (x) the flow past rectangular cylinders [15], (xi) the turbulent flow about a square tube [16], (xii) viscous flow past a primary circular cylinder is surrounded by smallmultiple control rods [17], (xiii) uniform flow past thorn (triangular solid) fixed to a square tube [18] and (xiv) the effects of corner modifications on the viscous flow and heat transfer about a square cylinder [19]. In recent times, powerful numerical methods like (i) immersed boundary methods are applied to heat flux boundary problems [20] and (ii) rotating hot cylinders in a cold square enclosure [21]. Though the Immersed boundary method is powerful, it is difficult to implement. Our present method is easy to apply. It is observed that the heat-transfer rate is very high in nanofluids and hence, nowadays, many researchers are investigating the heat transfer in nanofluids in different situations such as (i) mixed convection inside a vertical upward flow of nanofluid about a square tube using entropy generation [22] (ii) natural convection of $\mathrm{Cu}$-water nanofluid occupying eccentric annulus with uniform heat flux on the wall [23].

As in the above-mentioned cases, much literature is available on the problems related to the flow about a circular cylinder (review by Williamson [24]), flow and heat transfers about a square cavity. But less attention is paid to the study of flow past a circular cylinder in a cavity with suction applied on walls. Hence, in this paper, our aim is to examine the viscous fluid flow about a circular cylinder in a square cavity with suction on opposite walls.

\section{Statement of the problem}

The flow of viscous fluid past a cylinder in a square enclosure of uniform cross-section generated due to applied injection/suction at the opposite-side walls is considered. The Cartesian coordinate system is taken with origin at the bottom left corner of the cavity (enclosure) and $X$-axis and $Y$-axis are taken along the bottom and left walls. The cavity is of length $a_{0}$ (along $X$-axis) and height $b_{0}$ (along $Y$-axis). A cylinder of radius $r_{0}$ is kept in the centre of the cavity. Injection velocity $V_{1}$ at the left wall $X=0$ and suction velocity $V_{2}$ at the right wall $X=a_{0}$ are maintained. The flow is generated within the cavity due to the injection and suction applied at opposite-side walls as shown in Fig. 1.

Velocity of fluid particles satisfies no-slip condition on the top and bottom walls and on the cylinder. Again impermeability condition (normal velocity is zero) is taken on the cylinder. The permeability conditions, i.e. suction on the right wall and injection on the left wall are imposed. The temperature field satisfies (i) constant temperature on the impermeable sides $Y=0, Y=b_{0}$ and (ii) on the cylinder and (iii) uniform heat flux at permeable sides $X=0$ and $X=a_{0}$.

The equations of motion for the flow field are (the continuity equation, linear momentum equation and energy equation) given below:

$\nabla \cdot Q=0$
Fig. 1 Flow configuration past a cylinder in a square cavity

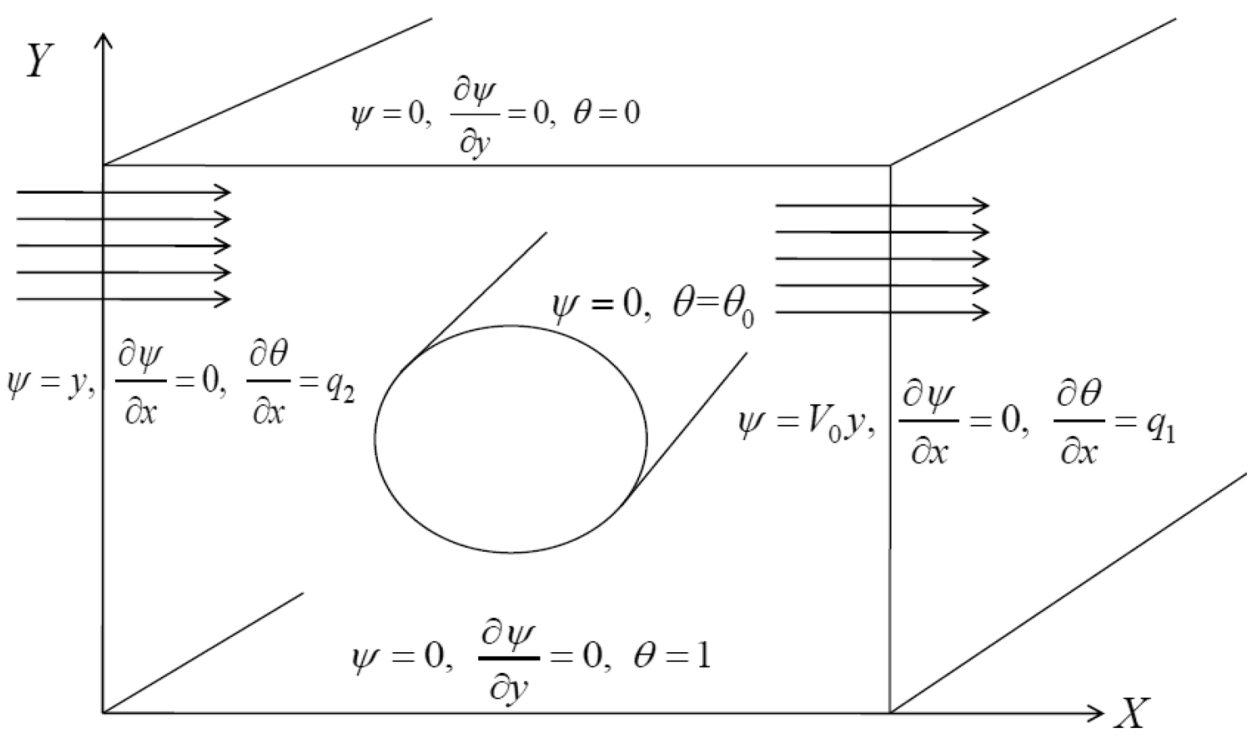


$\rho \frac{\mathrm{d} Q}{\mathrm{~d} t}=-\nabla P+\mu \nabla^{2} Q$

$\rho C_{\mathrm{p}} \frac{\mathrm{d} T}{\mathrm{~d} t}=k \nabla^{2} T$

Here, $Q$ is velocity of the fluid particle, $\rho$ is fluid density, $P$ is pressure, $\mu$ is the coefficient of viscosity, $T$ is the temperature, $k$ is thermal conductivity of the fluid and $C_{p}$ is heat capacity at constant pressure.

By geometry of the flow, the velocity is taken as $Q=(U$, V)

The non-dimensional scheme is introduced as below. Capital letters on LHS represent physical quantities and small letters on RHS represent non-dimensional quantities. The non-dimensional parameters, $\mathrm{Pe}=$ Peclet number, $y_{0}=$ geometric parameter, $V_{0}=$ suction parameter, are defined as below:

$X=a_{0} x, Y=a_{0} y, U=V_{1} u, V=V_{1} v, P=\rho V_{1}^{2} p, T-T_{1}=\theta\left(T_{2}-T_{1}\right)$

$\mathrm{Pe}=\frac{\rho C_{\mathrm{p}} V_{1} I}{k}, y_{0}=\frac{b_{0}}{a_{0}}, V_{0}=\frac{V_{2}}{V_{1}}$

For the flow, the Reynolds number is so small that the convective terms in Eq. (2) are neglected.

\subsection{Stream function}

Stream function $\psi$ is defined as below to satisfy Eq. (1).

$u=\frac{\partial \psi}{\partial y} \quad$ and $\quad v=-\frac{\partial \psi}{\partial x}$

By eliminating pressure from Eq. (2) and using (6), the equation for the stream function is obtained as

$\nabla^{4} \psi=0$

with boundary conditions:

$\frac{\partial \psi}{\partial x}=0$ on $x=0$ and on $x=1$ and $\psi=0$ on $I$

$\frac{\partial \psi}{\partial y}=1$ on $x=0$ and $\frac{\partial \psi}{\partial y}=V_{0}$ on $x=1$

$\frac{\partial \psi}{\partial x}=0$ on $y=0$ and on $y=y_{0}, \frac{\partial \psi}{\partial y}=0$ on $y=0$ and on $y=y_{0}$

By integrating these conditions, we get the conditions on $\psi$ as:

$$
\left.\begin{array}{rl}
\frac{\partial \psi}{\partial x} & =0 \text { on } x=0 \text { and } x=1 \\
\psi & =0 \text { on } y=0 \text { and } y=y_{0} \\
\psi & =y \text { on } x=0 \\
\psi & =V_{0} y \text { on } x=1 \\
\frac{\partial \psi}{\partial y} & =0 \text { on } y=0 \text { and } y=y_{0} \\
\psi & =0 \text { on the cylinder } \Gamma
\end{array}\right\}
$$

Equation (7) is solved under the conditions (8) by using FDM. The cavity is covered with uniform mesh of size $h$ with $(M-1)$ and $(N-1)$ intervals along $X$-direction and Y-direction. The bi-harmonic Eq. (7) is split into the following coupled equations [25]:

$\nabla^{2} \psi=-\zeta$

and $\nabla^{2} \zeta=0$

For the boundary conditions containing derivatives, central-difference scheme is used. The nodes are numbered as follows: $x_{1}$ and $y_{1}$ are nodes on the boundary $x=0$ and $y=0$, respectively. $x_{M}$ and $y_{N}$ are nodes on the boundary $x=1$ and $y=y_{0}$, respectively. $x_{2}, x_{3}, \ldots, x_{M-1}$ and $y_{2}, y_{3}, \ldots$, $y_{N-1}$ are points inside the computational domain.

Thus at $x=0$, we have $\psi(0, y)=y$. i.e., at $i=1$,

$$
\psi_{1, j}=(j-1) h \text { for } j=1,2, \ldots, N
$$

at $x=1$, we have $\psi(1, y)=V_{0} y$. i.e.,

$$
\text { at } i=M, \psi_{M, j}=V_{0}(j-1) h \text { for } j=1,2, \ldots, N
$$

at $y=0$, we have. i.e., at $j=1, \psi(x, 0)=0$

$\psi_{i, 1}=0$ for $i=2,3, \ldots, M-1$

at $y=y_{0}$, we have $\psi\left(x, y_{0}\right)=0$. i.e., at $j=N$,

$\psi_{i, N}=0$ for $i=2,3, \ldots, M-1$

The boundary values of $\zeta$ in terms of $\psi$ are derived as below. The vorticity on $x=0$ [i.e. at $(1, j)]$ is assumed as:

$$
\begin{aligned}
\zeta_{1, j}= & -\left(\frac{\partial^{2} \psi}{\partial x^{2}}+\frac{\partial^{2} \psi}{\partial y^{2}}\right)_{1, j}=\alpha_{1} \psi_{2, j-1} \\
& +\alpha_{2} \psi_{2, j}+\alpha_{3} \psi_{2, j+1}+\alpha_{4} \psi_{3, j}+\alpha_{5}\left(\frac{\partial \psi}{\partial x}\right)_{1, j}
\end{aligned}
$$

By expanding the functions in Taylor's series, we have 


$$
\begin{gathered}
\psi_{2, j \pm 1}=\psi_{1, j}+h\left(\frac{\partial \psi}{\partial x}\right)_{1, j} \pm h\left(\frac{\partial \psi}{\partial y}\right)_{1, j} \\
+\frac{h^{2}}{2}\left(\frac{\partial^{2} \psi}{\partial x^{2}}\right)_{1, j}+\frac{h^{2}}{2}\left(\frac{\partial^{2} \psi}{\partial y^{2}}\right)_{1, j}+O\left(h^{3}\right) \\
\psi_{2, j}=\psi_{1, j}+h\left(\frac{\partial \psi}{\partial x}\right)_{1, j}+\frac{h^{2}}{2}\left(\frac{\partial^{2} \psi}{\partial x^{2}}\right)_{1, j}+O\left(h^{3}\right) \\
\psi_{3, j}=\psi_{1, j}+2 h\left(\frac{\partial \psi}{\partial x}\right)_{1, j}+\frac{(2 h)^{2}}{2}\left(\frac{\partial^{2} \psi}{\partial x^{2}}\right)_{1, j}+O\left(h^{3}\right)
\end{gathered}
$$

Using these expressions, Eq. (15) becomes

$$
\begin{aligned}
& -\left(\frac{\partial^{2} \psi}{\partial x^{2}}+\frac{\partial^{2} \psi}{\partial y^{2}}\right)_{1, j}=\left(\alpha_{1}+\alpha_{2}+\alpha_{3}+\alpha_{4}\right) \psi_{1, j} \\
& +\left[\left(\alpha_{1}+\alpha_{2}+\alpha_{3}+2 \alpha_{4}\right) h+\alpha_{5}\right]\left(\frac{\partial \psi}{\partial x}\right)_{1, j} \\
& +\left(\alpha_{3}-\alpha_{1}\right) h\left(\frac{\partial \psi}{\partial y}\right)_{1, j} \\
& +\left[\left(\alpha_{1}+\alpha_{2}+\alpha_{3}+4 \alpha_{4}\right) \frac{h^{2}}{2}\right]\left(\frac{\partial^{2} \psi}{\partial x^{2}}\right)_{1, j} \\
& +\left(\alpha_{1}+\alpha_{3}\right) \frac{h^{2}}{2}\left(\frac{\partial^{2} \psi}{\partial y^{2}}\right)_{1, j}
\end{aligned}
$$

The values for $a$ 's are obtained by equating the coefficients of like terms on the both sides. Then, Eq. (15) reduces to

$\zeta_{1, j}=\frac{1}{h^{2}}\left(-\psi_{2, j-1}+\frac{8}{3} \psi_{2, j}-\psi_{2, j+1}-\frac{2}{3} \psi_{3, j}\right)$ for $j=2,3, \ldots N$

where $\left(\frac{\partial \psi}{\partial x}\right)_{1, j}=0$ from the boundary condition (8).

Similarly, the expressions for vorticity at the remaining boundaries as:

$\zeta_{S}=\frac{\left(\psi_{G}-\psi_{S}\right)}{D}$

Here, $G$ is the point on the normal which cuts either vertical or horizontal grid line in the flow region, $S$ is the point on the cylinder and $D$ is the distance between the points $G$ and $S$.

Equation (10) numerically will be solved using Liebmann's iterative formula:

$\zeta_{i, j}=\frac{1}{4}\left(\zeta_{i-1, j}+\zeta_{i+1, j}+\zeta_{i, j-1}+\zeta_{i, j+1}\right)$

The points on the boundary of the cylinder are obtained when the horizontal-vertical grid lines intersect the body surface. These points in general do not coincide with the grid points. Hence, the formula (21) cannot be used at points on the cylinder and at points in the immediate neighbourhood of the cylinder. Hence, the difference Eq. (21) has to be generalized for an arbitrary grid (as shown in Fig. 2 ) in which the point $(i, j)$ is at different distances $a, b, c$ and $d$ from its four neighbouring points.

Here $\zeta_{a}=\zeta_{i-1, j}, \zeta_{b}=\zeta_{i+1, j}, \zeta_{c}=\zeta_{i, j-1}$, and $\zeta_{d}=\zeta_{i, j+1}$. Now, we approximate the left hand side of $(10)$ at $(i, j)$ as the linear function as below:

$\left(\frac{\partial^{2} \zeta}{\partial x^{2}}+\frac{\partial^{2} \zeta}{\partial y^{2}}\right)_{i, j}=\alpha_{0} \zeta_{i, j}+\alpha_{a} \zeta_{a}+\alpha_{b} \zeta_{b}+\alpha_{c} \zeta_{c}+\alpha_{d} \zeta_{d}$

where $a$ 's are coefficient to be determined by procedure taken for Eq. (15).

$\zeta_{i-1, j}=\zeta_{a}=\zeta_{i, j}-a\left(\frac{\partial \zeta}{\partial x}\right)_{i, j}+\frac{a^{2}}{2}\left(\frac{\partial^{2} \zeta}{\partial x^{2}}\right)_{i, j}-O\left(a^{3}\right)$

$\zeta_{i, j+1}=\zeta_{d}=\zeta_{i, j}+d\left(\frac{\partial \zeta}{\partial y}\right)_{i, j}+\frac{d^{2}}{2}\left(\frac{\partial^{2} \zeta}{\partial y^{2}}\right)_{i, j}+O\left(d^{3}\right)$

$\underline{\text { and so forth. Substitution of these equations in (22) after }}$

on $x=1, \quad \zeta_{M, j}=\frac{1}{h^{2}}\left(-\psi_{M-1, j-1}+\frac{8}{3} \psi_{M-1, j}-\psi_{M-1, j+1}-\frac{2}{3} \psi_{M-2, j}\right)$ for $j=2,3, \ldots N$

on $y=0, \quad \zeta_{i, 1}=\frac{1}{h^{2}}\left(-\psi_{i-1,2}+\frac{8}{3} \psi_{i, 2}-\psi_{i+1,2}-\frac{2}{3} \psi_{i, 3}\right)$ for $i=2,3, \ldots M$

on $y=y_{0}, \quad \zeta_{i, N}=\frac{1}{h^{2}}\left(-\psi_{i-1, N-1}+\frac{8}{3} \psi_{i, N-1}-\psi_{i+1, N-1}-\frac{2}{3} \psi_{i, N-2}\right)$ for $i=2,3, \ldots M$

In the above equations, the derivative boundary conditions in (8) have been employed.

Along with these boundary conditions, we have $\zeta$ on the cylinder is given by Thom [26] rearrangement and neglecting the cubic and higher-order terms, we get $\left(\frac{\partial^{2} \zeta}{\partial x^{2}}+\frac{\partial^{2} \zeta}{\partial y^{2}}\right)_{i, j}=\left(\alpha_{0}+\alpha_{a}+\alpha_{b}+\right.$ 


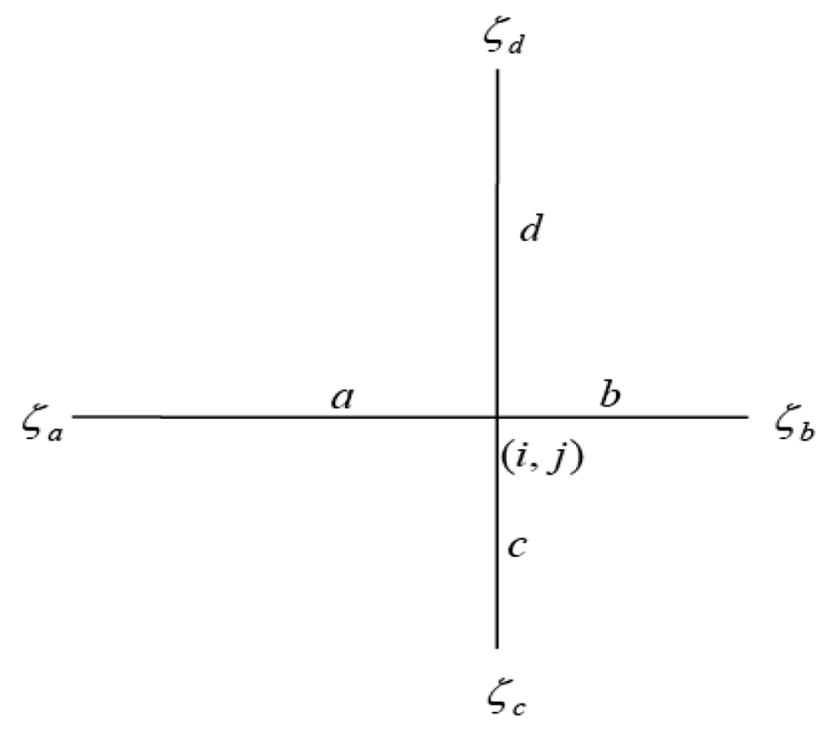

Fig. 2 Evaluation of $\zeta_{i, j}$

$\left.\alpha_{c}+\alpha_{d}\right) \zeta_{i, j}+\left(b \alpha_{b}-a \alpha_{a}\right)\left(\frac{\partial \zeta}{\partial x}\right)_{i, j}+\left(d \alpha_{d}-c \alpha_{c}\right)\left(\frac{\partial \zeta}{\partial y}\right)_{i, j}+$ $\frac{1}{2}\left(a^{2} \alpha_{a}+b^{2} \alpha_{b}\right)\left(\frac{\partial^{2} \zeta}{\partial x^{2}}\right)_{i, j}+\frac{1}{2}\left(c^{2} \alpha_{c}+d^{2} \alpha_{d}\right)\left(\frac{\partial^{2} \zeta}{\partial y^{2}}\right)_{i, j}$ The coefficients of like terms on both sides give the values of a's as below:

$\alpha_{0}=-2\left(\frac{1}{a b}+\frac{1}{c d}\right), \alpha_{a}=\frac{2}{a(a+b)}, \alpha_{b}=\frac{2}{b(a+b)}$

$\alpha_{c}=\frac{2}{c(c+d)}, \alpha_{d}=\frac{2}{d(c+d)}$

Vanishing of the right hand side of (22) gives the desired difference approximation of the Laplace equation:

$\zeta_{i, j}=\left[\frac{\zeta_{a}}{a(a+b)}+\frac{\zeta_{b}}{b(a+b)}+\frac{\zeta_{c}}{c(c+d)}+\frac{\zeta_{d}}{d(c+d)}\right] /\left(\frac{1}{a b}+\frac{1}{c d}\right)$

for $i=2,3, \ldots, M-1$ and $j=2,3, \ldots, N-1$

It can easily be shown that (23) is equivalent to (21) when $a=b=c=d=h$.

In the similar manner we solve Eq. (9) numerically using

$\psi_{i, j}=\left[\frac{\psi_{a}}{a(a+b)}+\frac{\psi_{b}}{b(a+b)}+\frac{\psi_{c}}{c(c+d)}+\frac{\psi_{d}}{d(c+d)}+\frac{\zeta_{i, j}}{2}\right] /\left(\frac{1}{a b}+\frac{1}{c d}\right)$

for $i=2,3, \ldots, M-1$ and $j=2,3, \ldots, N-1$

The values of vorticity at walls are derived in (16)-(20) which will enable us to solve (10) when $\psi$ is known at the interior points. However, the determination of $\psi$ from (9) depends on the values of vorticity within the domain.
Thus, $\psi$ and $\zeta$ are coupled. Hence, an iterative scheme is built up to solve them.

For initial guess, first a stationary state is assumed such that $\psi=0$ everywhere in the domain. Based on these values of $\psi$, the values of $\zeta$ on the walls are computed from (16)-(20), thus at the boundary, vorticity is initially generated. This concentrated vorticity at the walls starts to diffuse into the interior of domain, resulting in a temporary vorticity distribution which is the solution of (10) that is satisfying the boundary conditions. Now, this vorticity causes a modification to $\psi$. In this way, the first iteration is completed. In the next iteration, based on $\psi$, the boundary values of $\zeta$ are recomputed. To get a next iteration values for $\psi$ and $\zeta$, the same procedure is repeated. At every grid point, the difference between successive iteration values of $\zeta$ is recorded as the local error during each iteration and the sum of absolute errors at all grid points is called ERZETA. Similarly, ERPSI stands for the corresponding sum of absolute errors of $\psi$. Iteration is terminated when both ERPSI and ERZETA are smaller than a small positive value (EPSLON $=10^{-4)}$. When the desired accuracy is reached, the solution is then considered to be satisfactory.

\subsection{Temperature}

The energy Eq. (3) is reduced to the non-dimensional form as below:

$\nabla^{2} \theta=P e\left(u \frac{\partial \theta}{\partial x}+v \frac{\partial \theta}{\partial y}\right)$

Temperature is subjected to the boundary conditions that (i) the sides $y=0, y=y_{0}$ and on cylinder is maintained at constant temperatures and (ii) the other sides are supplied with constant heat or constant heat flux. The conditions are given explicitly as

$\left.\begin{array}{rl}\frac{\partial \theta}{\partial x} & =q_{1} \text { on } x=1 \\ \theta & =0 \text { on } y=y_{0} \\ \theta & =1 \text { on } y=0 \\ \frac{\partial \theta}{\partial y} & =q_{2} \text { on } x=0 \\ \theta & =\theta_{0} \text { on cylinder }\end{array}\right\}$

Let $u_{i, j}=\left(\frac{\partial \psi}{\partial y}\right)_{i, j}=\frac{\psi_{i, j+1}-\psi_{i, j-1}}{2 h}=f_{2}$ and $v_{i, j}=-\left(\frac{\partial \psi}{\partial x}\right)_{i, j}$ $=-\frac{\psi_{i+1, j}-\psi_{i-1, j}}{2 h}=-f_{1}$

Proceeding as in the stream function and vorticity function, Eq. (25) can be generalized as:

$\theta_{i, j}=\left[\frac{\left(2+a f_{2} P e\right) \theta_{a}}{a(a+b)}+\frac{\left(2-b f_{2} P e\right) \theta_{b}}{b(a+b)}+\frac{\left(2-c f_{1} P e\right) \theta_{c}}{c(c+d)}+\frac{\left(2+d f_{1} P e\right) \theta_{d}}{d(c+d)}\right] / 2\left(\frac{1}{a b}+\frac{1}{c d}\right)$ 

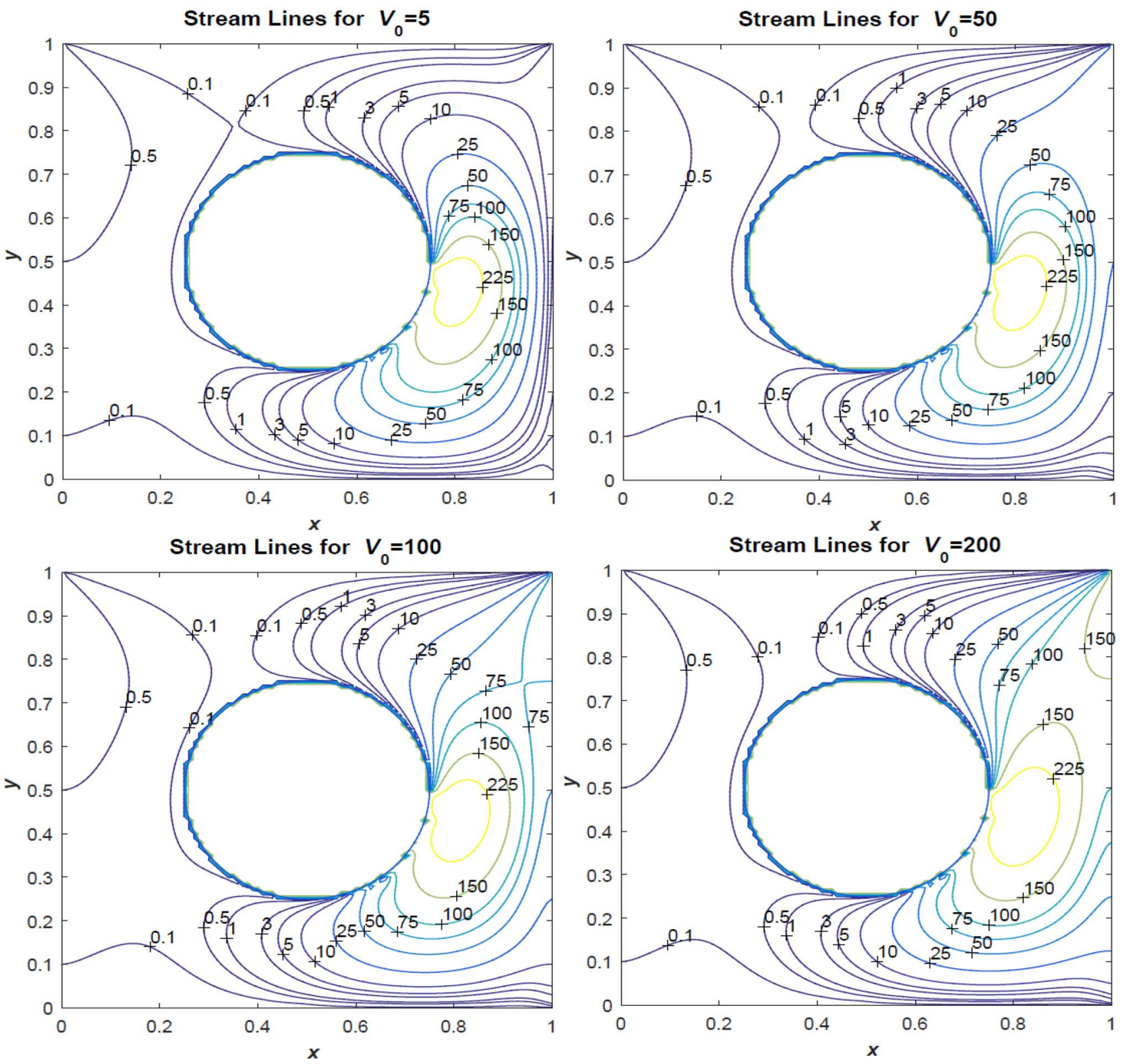

Fig. 3 Streamlines for different values of $V_{0}$

for $i=2,3, \ldots, M$ and $j=2,3, \ldots, N$

The boundary conditions in (26) are now expressed as:
As in stream function and vorticity function in Eq. (27) subjected to the boundary conditions (28) will be solved for the temperature in the cavity.

at $x=0, \quad \frac{\partial \theta}{\partial x}=q_{2}$ which implies that $\theta_{1, j}=\frac{1}{3}\left[4 \theta_{2, j}-\theta_{3, j}-2 h q_{2}\right]$ for $j=1,2, \ldots, N$

at $y=0, \quad \theta=1$ which implies that $\theta_{i, 1}=1$ for $i=2,3, \ldots, M-1$

at $y=y_{0}, \quad \frac{\partial \theta}{\partial x}=q_{1}$ which implies that $\theta_{M, j}=\frac{1}{3}\left[4 \theta_{M-1, j}-\theta_{M-2, j}-2 h q_{1}\right]$ for $j=1,2, \ldots, N$

and on the cylinder $\theta_{i, j}=\theta_{0}=0.5$ where $\theta_{0}$ is the reference temperature 

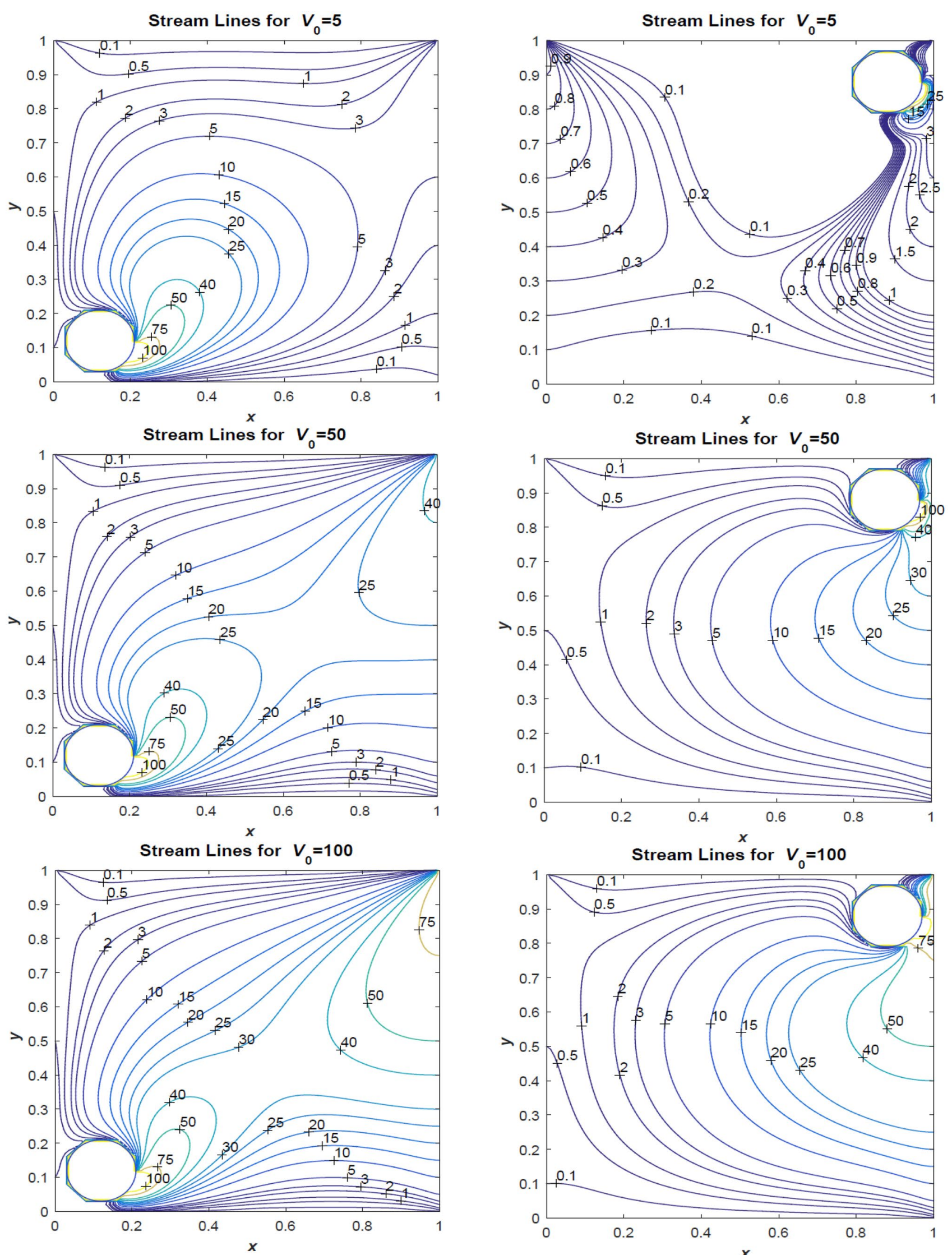

Fig. 4 Streamlines for different values of $\left(V_{0}=5,50,100\right)$ when cylinder is at lower left corner of the cavity

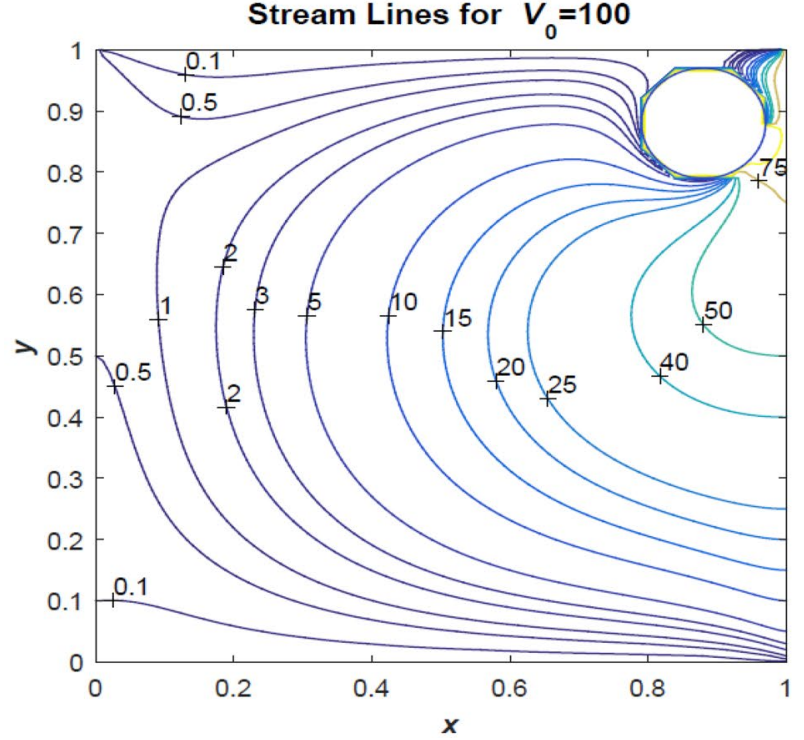

Fig. 5 Streamlines for different values of $\left(V_{0}=5,50,100\right)$ when cylinder is at top right corner of the cavity 


\section{Results}

We have obtained the values of stream function and temperature for step length $h=0.01$. The accuracy near to 4 decimal places is obtained by taking 1000 iterations. Here, the important steps involved in computation are to calculate (1) $a, b, c, d$ values adjacent to the cylinder and (2) $h$ the distance between $G$ and $S$ in Eq. (20). Here, $S$ is a point on the cylinder and $G$ is a point on the grid line obtained by intersection of normal with the grid. These are explained in the "Appendix".

Streamlines The stream lines generated due to injection at the left wall and suction at the right wall are shown in Figs. 3, 4 and 5. The figures are drawn for different suction parameter values $V_{0}$. We observe from Fig. 3 that when the cylinder is at middle of the cavity, as suction parameter $V_{0}$ increases, the region of high stream values increases at the right end of the cylinder and the stream lines with low values (for example $\psi=0.1$ ) are separated. Again, as $V_{0}$ increases, a second circulation region is formed at the top left corner. For small values of $V_{0}$, the stream lines with high values are nearly parallel to the right wall.

From Fig. 4, when the cylinder is bottom left corner, for small suction $V_{0}=5$, stream lines having small values $<1$ are parallel to the top wall and having high values $>1$ are forming loops behind the cylinder and as $V_{0}$ increases, these loop lines open to the top side and bifurcate from the region of looping. The presence of cylinder effects
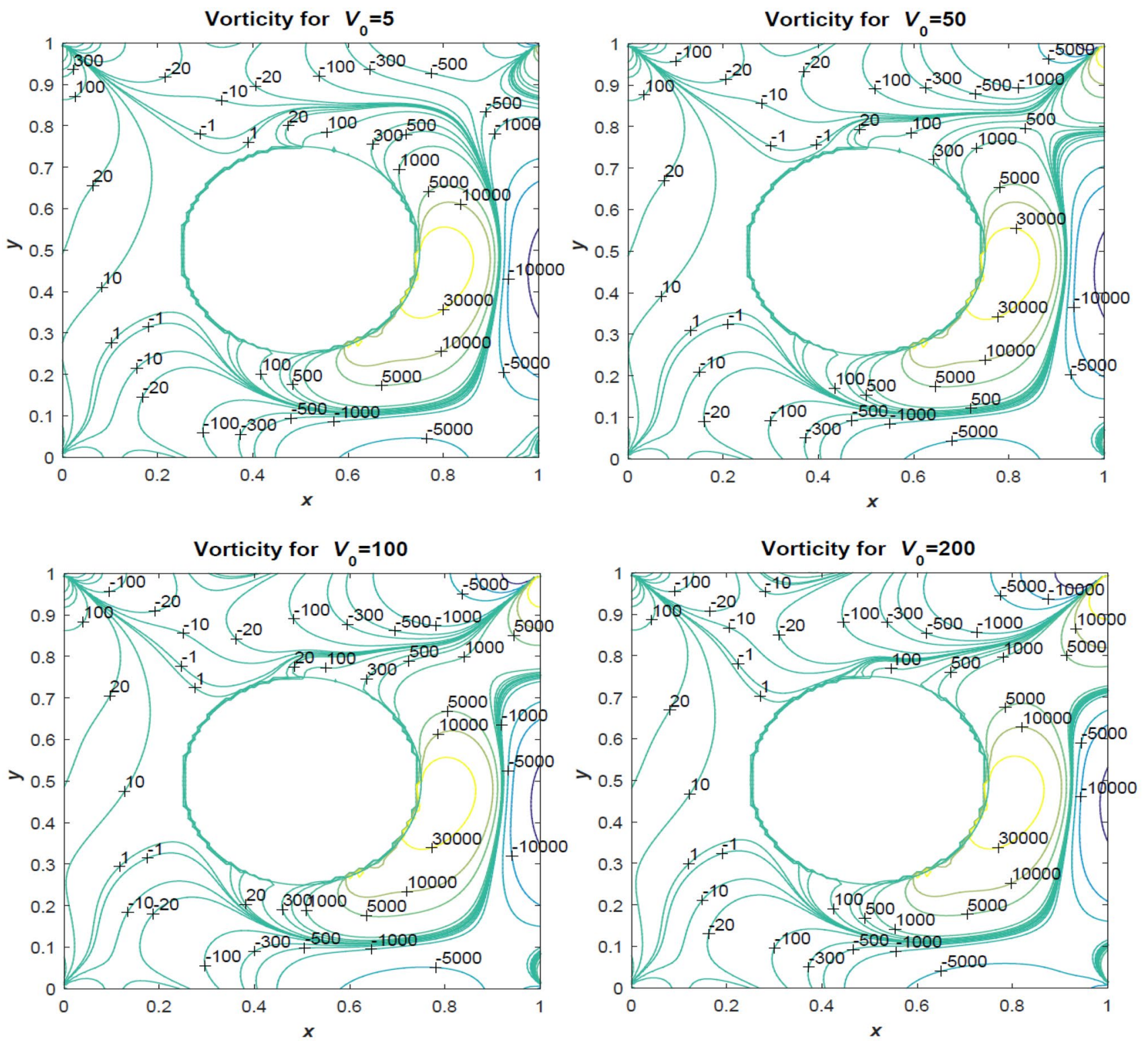

Fig. 6 Vorticity contours for different values of $V_{0}$ 

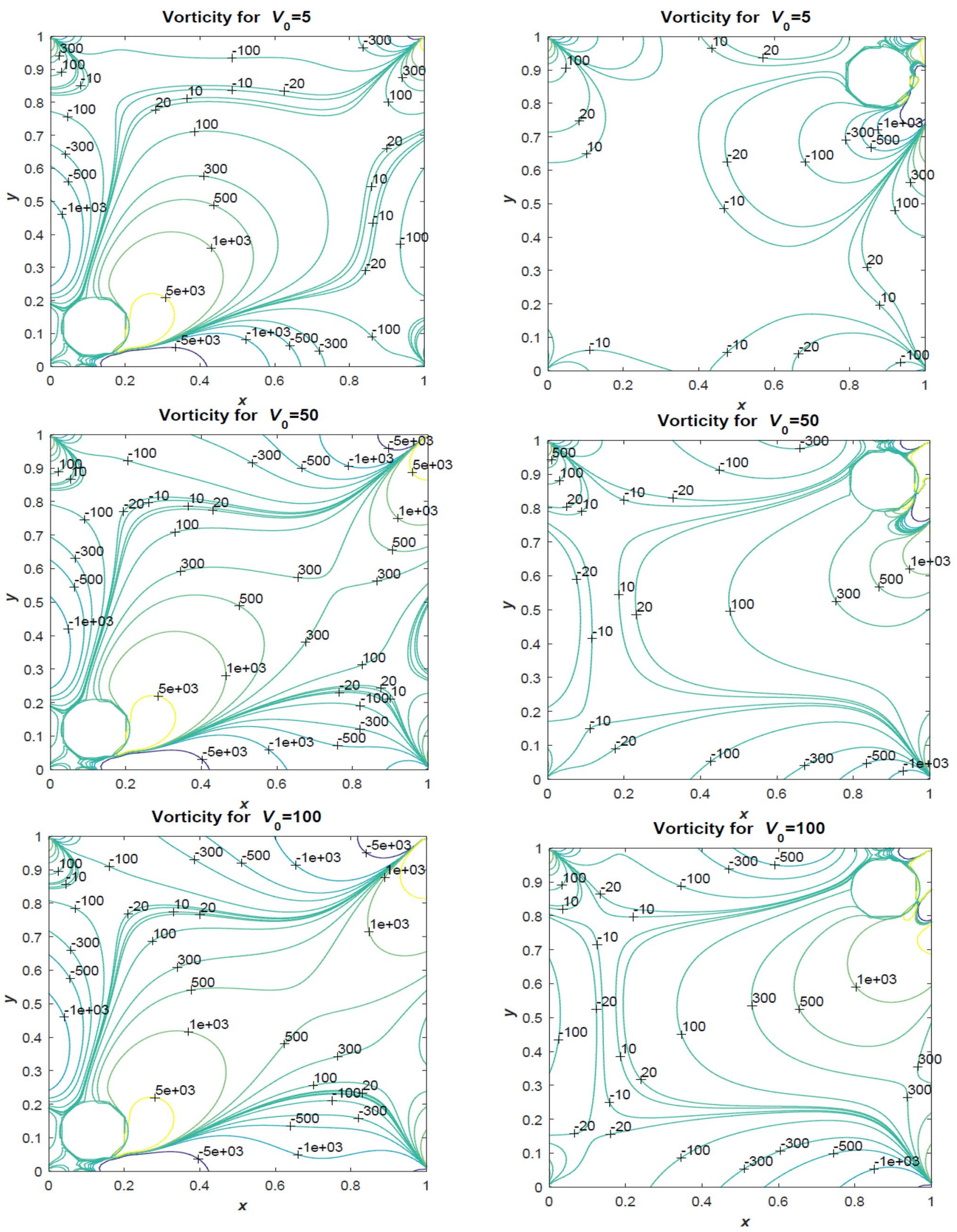

Fig. 7 Vorticity contours for different values of $\left(V_{0}=5,50,100\right)$ when cylinder is at lower left corner of the cavity

Fig. 8 Vorticity contours for different values of $\left(V_{0}=5,50,100\right)$ when cylinder is at top right corner of the cavity 

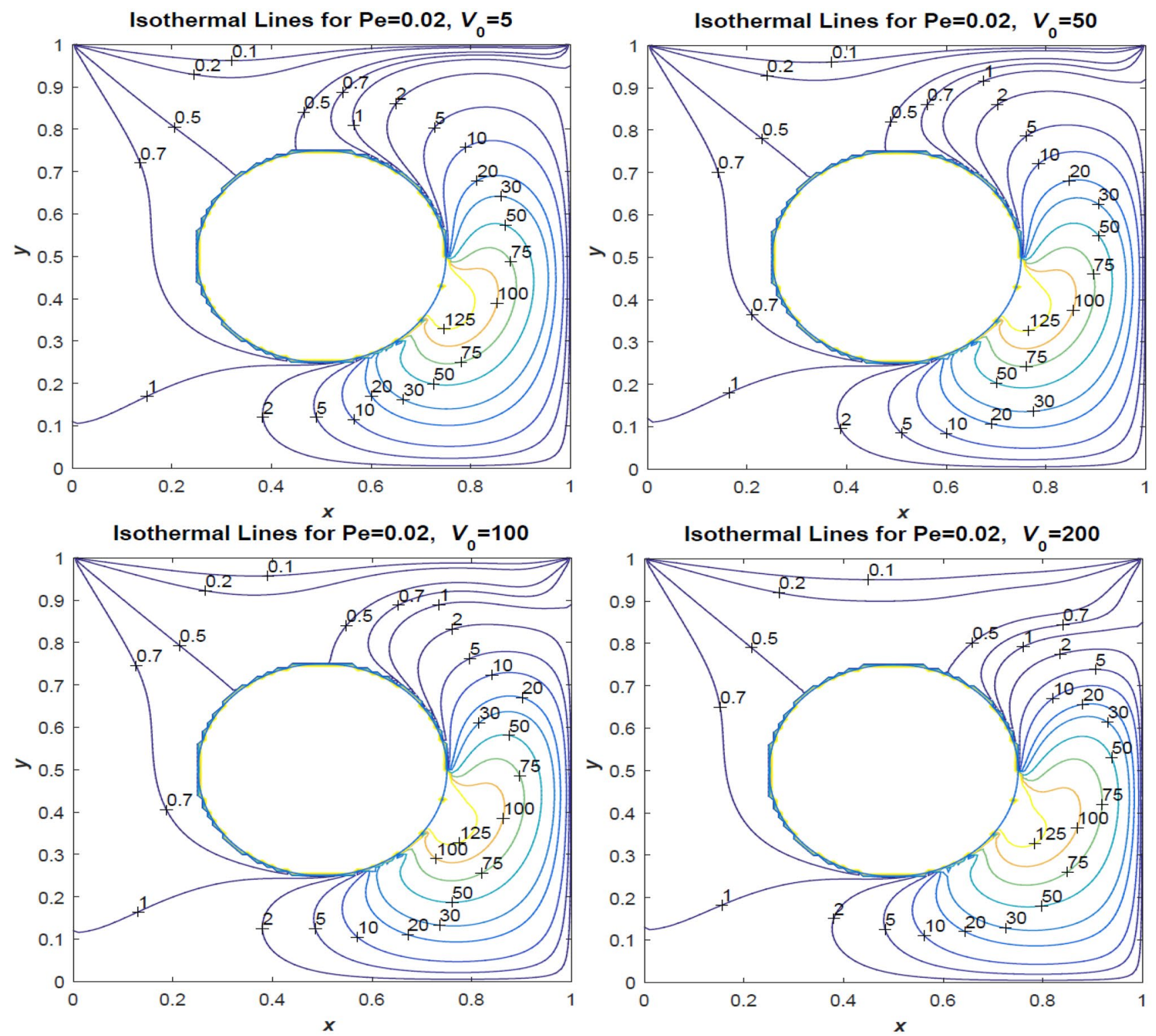

Fig. 9 Isothermal lines for different values of $V_{0}$ at $\mathrm{Pe}=0.02$

entire region dominating suction. As suction parameter increases, the effect of suction spreads near to the cylinder and the values of stream lines away from the cylinder also increase.

It is important to note that even though cylinder is small placed at left corner effects the entire flow dominating the suction.

From Fig. 5 when the cylinder is at the top right corner, we find that for any value of suction parameter, circulation loops are not found. It is because there is no space behind the cylinder to form wakes. For small values of suction, the stream lines take small values and the flow is in four distinct regions. But when suction increases, all distinct regions merge to a single region. But for any value of $V_{0}$, we can find flow regions with $\psi<1$ and $\psi>1$. As $V_{0}$ increases, the region with $\psi>1$ occupies the most of the space.

It is important to note that when the cylinder is at the top right corner, no circulation wakes are found.

The circulation wakes behind the cylinder are best observed when it is in the bottom left corner.

When there is no cylinder, the steam line pattern is shown in Fig. 13. For high suction, when the smaller cylinder is on the top corner, the flow is similar to the flow without cylinder.

Vorticity Contours The vorticity contours generated due to injection at the left wall and suction at the right wall for different suction parameters $V_{0}$ are shown in Figs. 6,7 and 
8. From Fig. 6 , we observe that as $V_{0}$ increases, when the cylinder is middle of the cavity, the circulation of vortex lines on the right side of cylinder increases and opens to the right top corner. The values of vorticity are very high at the right wall and in the circulation region of vorticity. Outside of the circulation region, vorticity takes negative values except at top left region.

From Fig. 7, when a small cylinder is placed at the bottom left corner, for small values of suction, the circulation region above the cylinder spreads to the top corner. High values of vorticity are found on the left wall which is opposite to the above case (when the cylinder is in the middle). As $V_{0}$ increases, circulation region is confined to a small region near to the cylinder.

From Fig. 8, when a small cylinder is placed at the top right corner, for small suction parameter circulation region below the cylinder is small in the region and this region spreads in the entire cavity when suction increases. Here, we find high negative values of vorticity outside the circulation region at the top and bottom walls.

Temperature field Figures. 9, 10, 11 and 12 display the nature of temperature contours. Figure 9 shows the isothermal lines for different values of $V_{0}$ at $\mathrm{Pe}=0.02$. We observe that the line $\theta=1$ divides the entire region into two parts: the region in which $\theta$ is less than 1 and the region in which $\theta$ is more than 1 . As $V_{0}$ increases, the region with $\theta<1$ increases and spreads to the top wall. The region with $\theta>1$ forms a circular loops. As Peclet number $P e$ increases, the temperature in the region $\theta>1$ also inc reases.

Figure 10 shows the isothermal lines for different values of $V_{0}$ at $\mathrm{Pe}=0.02$ when a small cylinder is placed at the lower left corner. The region $\theta<1$ is at the top of the cylinder in a small area for all suction values. Near the right side of the cylinder, we find very high temperature.

Figure 11 shows the isothermal lines for different values of $V_{0}$ at $\mathrm{Pe}=0.02$ when the cylinder is placed on the top right corner. We observe that as $V_{0}$ increasing, the region $\theta<1$ is decreasing.

Figure 12 shows the isothermal lines for different values of $P e$ at $V_{0}=10$. We observe that Peclet number does not show much effect on the isothermal lines.

From Figs. 14 and 12, we observe that temperature lines are similar when cylinder becomes smaller and smaller. We observe that because of cylinder placed in the cavity the temperature increases drastically in all cases in comparison with without cylinder.

\section{Discussions}

Here, we have used standard 5-point formula to solve for stream function, vorticity and temperature. This scheme is second-order accurate. Powerful techniques like immersed boundary method are recently developed for higher-order methods. But for implementation, they are not easy to handle, whereas this standard 5-point formula is easy to implement and straight forward. To analyse the results, this formula is sufficient and accurately enough.

Stream lines and Vorticity lines We consider that fluid enters the cavity by injection at the left wall and leaves the chamber by suction at the right wall. Hence, fluid flows from the left wall to right wall. Therefore, if a cylinder is placed in the cavity, wakes are expected behind the left side of the cylinder. The same we can find in Figs. 3, 4 and 5 for stream lines and in Figs. 6, 7 and 8 for vorticity lines. Relatively big cylinder at the centre of the cavity is considered in Figs. 3 and 6. In these figures, for stream lines or vorticity lines, it is natural to find circulations behind left side of the cylinder. If we consider stream line with value 0.1 , for $V_{0}=5$, (Fig. 3) the stream lines almost encloses the cylinder. When $V_{0}$ increases to 50, 100 and 200, this line opens up and deviates from the cylinder. This is because when suction/injection increases, more fluid is pushed into the cavity and creates strong wakes behind the cylinder (on the opposite side of the flow). In the flow pattern (when the cylinder is in the middle of the cavity Figs. 3 and 6), we do not observe much change. But the values of the function within the circulations increase.

When the small cylinder is placed at the left bottom corner Fig. 4, we can find circulations behind the cylinder. When injection increases, the values of the circulations increase and a second zone of circulations near to right wall is also found. This is due to injection rate is more than suction rate. When the cylinder is placed at the top right corner (Fig. 5), there is no much region behind the cylinder and hence wakes behind the cylinder are also weak. Hence, when $V_{0}$ is high, the flow pattern is similar to free stream flow (Fig. 13) when there is no cylinder. A similar interpretation can be given to vorticity pattern.

Temperature distribution The top and bottom walls of the cavity are maintained at non-dimensional temperatures 0 and 1 . The left and right walls are maintained at constant heat flux due to injection and suction. Hence, in the isothermal lines, we observe temperatures near to 

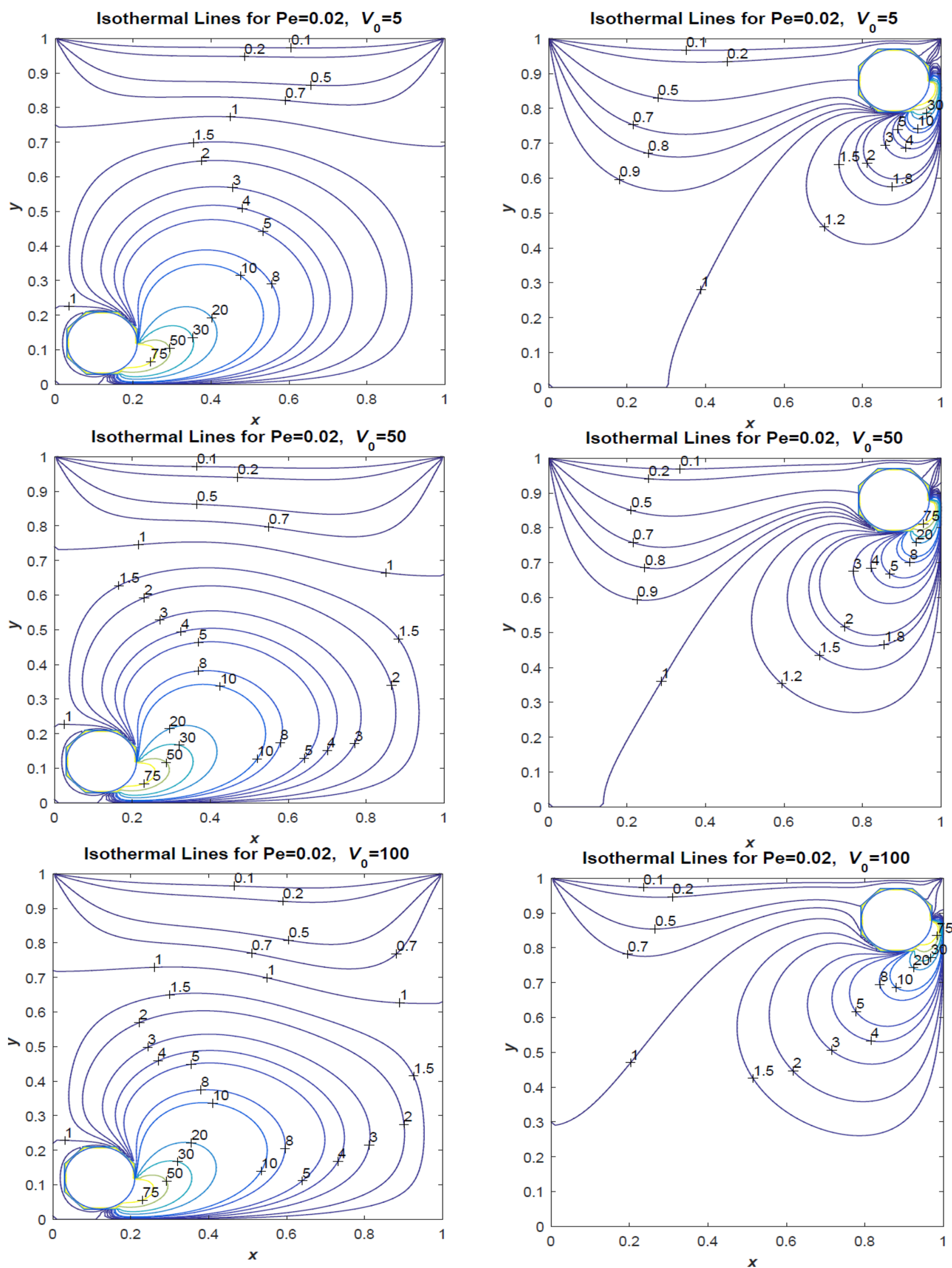

Fig. 10 Isothermal lines for different values of $\left(V_{0}=5,50,100\right)$ at $\mathrm{Pe}=0.02$ when cylinder is at lower left corner of the cavity

Fig. 11 Isothermal lines for different values of $\left(V_{0}=5,50,100\right)$ at $\mathrm{Pe}=0.02$ when cylinder is at top right corner of the cavity 

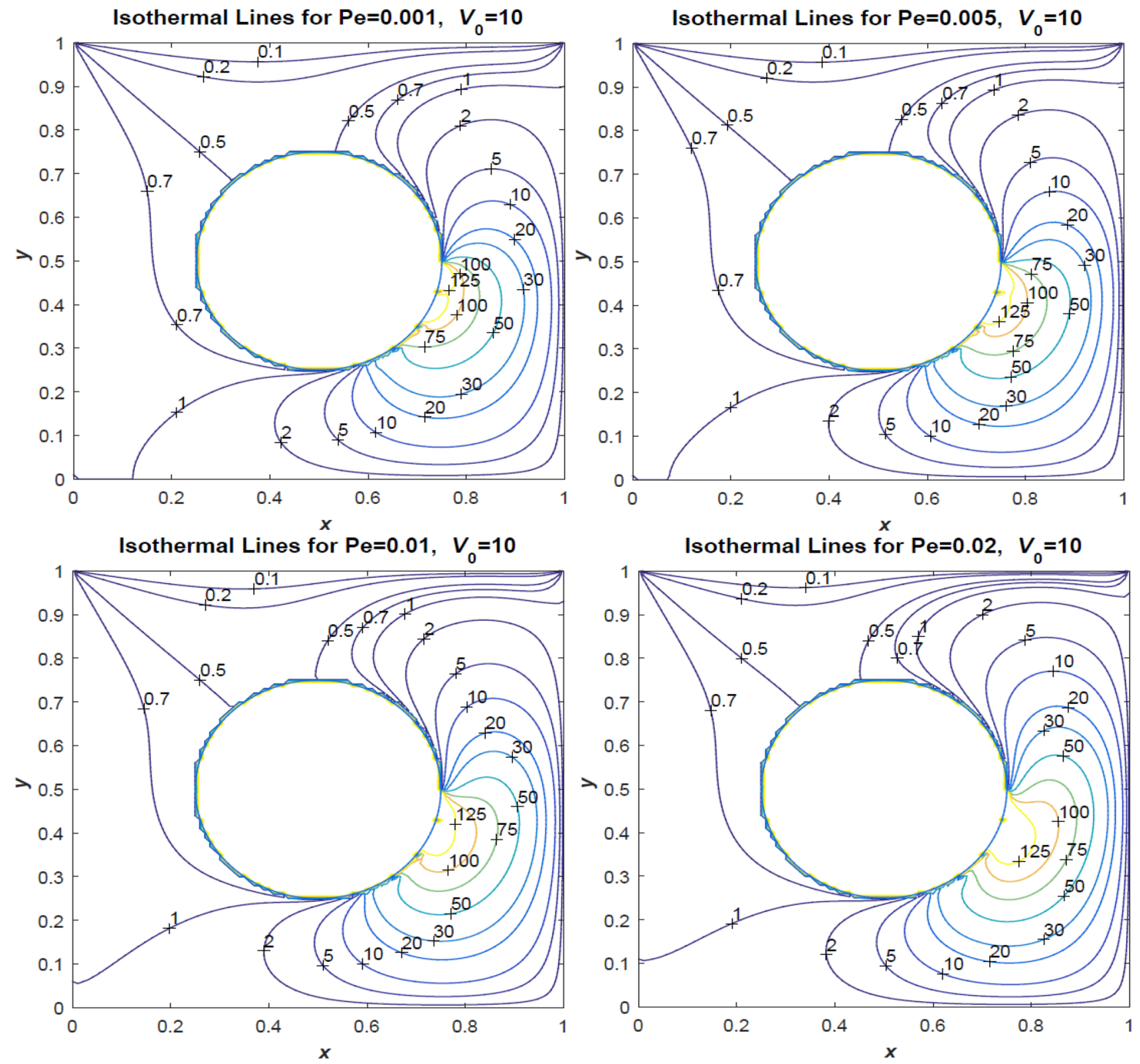

Fig. 12 Isothermal lines for different values of $P e$ at $V_{0}=10$

zero on the top and temperatures near to 1 near to the bottom wall. Due to wakes formed behind the cylinder, in these regions, there will be energy dissipation and hence high temperature is generated. Hence, the temperature contours are similar to stream line contours. Here, we can find an interesting observation that the entire temperature region is divided into two: one with temperature less than one $(\theta<1)$ and one with temperature more than one $(\theta>1)$. This is due to constant temperature and heat flux conditions on the walls.
On comparing the temperature profiles of Fig. 14 with other Figs. 9, 10, 11 and 12, we observe that Fig. 14 is not similar to any other figures for temperatures. Which means even if the cylinder is small, it enhances the heat transfer to a considerable extent. This observation is in contrast to stream line pattern where, at high $V_{0}$ for small cylinder placed at the top corner, the flow pattern is similar to the free stream pattern where there is no cylinder in the cavity. 


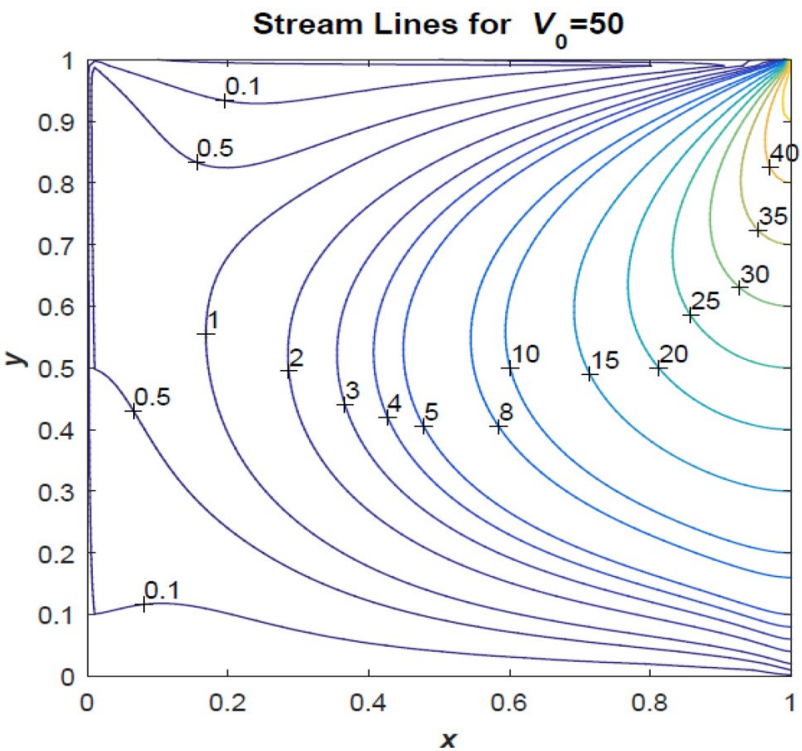

Fig. 13 Streamlines for $V_{0}=50$ when cylinder is absent in the cavity

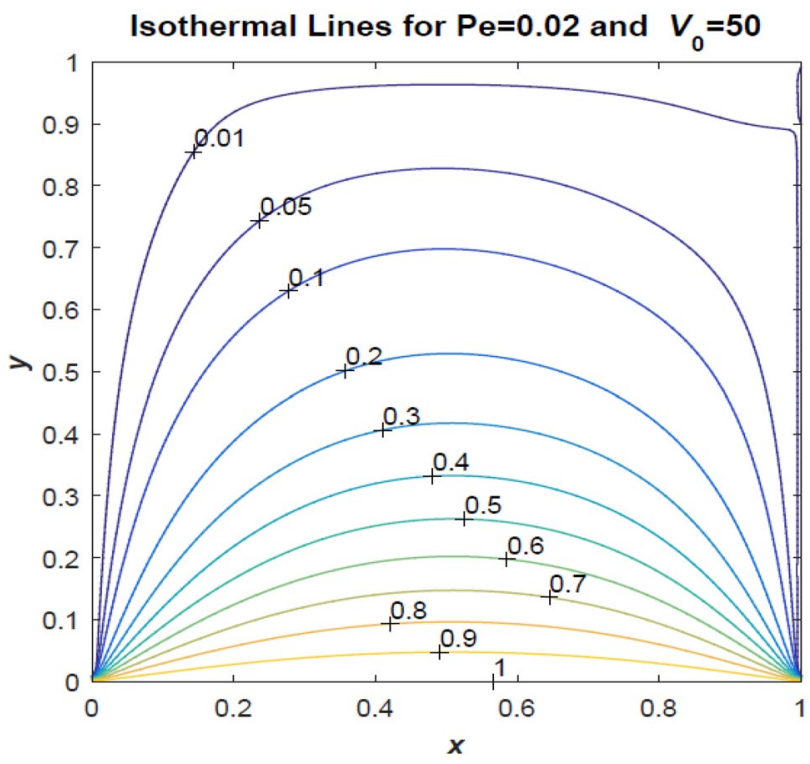

Fig. 14 Isothermal lines for $\mathrm{Pe}=0.05$ and $V_{0}=50$ when cylinder is absent in the cavity

\section{Conclusions}

From the above observations, we conclude that

1. As suction parameter $V_{0}$ increases, stream line pattern will not change much when the cylinder is in the middle of the cavity. But when it is at the top right corner, suction effect is very much high.
2. Due to the boundary conditions on temperature, the entire temperature zone is divided into two regions $\theta<1$ and $\theta>1$.

3. As suction parameter $V_{0}$ increases, region $\theta<1$ spreads to suction wall.

4. The temperature increases drastically within the cavity, when a cylinder is kept in the cavity, i.e. introduction of an object (here cylinder) into the cavity will enhance the heat transfer.

\section{Compliance with ethical standards}

Conflict of interest The authors declare that they have no conflict of interest

\section{Appendix}

Let the grid along $x$-direction and $y$-direction cut the circle at $Q_{1}$ and $Q_{2}$, respectively. The normals on the circle at $Q_{1}$ and $Q_{2}$ cut the grid at $G_{1}$ and $G_{2}$ along $y$-direction and $x$-direction. Refer the Fig. 15.

Let $S$ be the circle. The interval $[0,1]$ along $x$-direction is divided into $M-1$ intervals. The interval $\left[0, y_{0}\right]$ along $y$-direction is divided into $N-1$ intervals. The grid points along $x$-direction and $y$-direction are indicated by $i$ and $j$. we take $M$ and such that $h=\frac{1}{M-1}=\frac{y_{0}}{N-1}$ or $y_{0}=\frac{N-1}{M-1} \cdot i=j=1$ and $i=M, j=N$ are on the walls of the cavity. Any point $P$ has coordinates $\left(x^{*}, y^{*}\right)$ and has the corresponding grid

\section{$S=(i, j)=$ a point on the surface \\ $\mathrm{G}=$ normal at $\mathrm{S}$ cuting the grid}

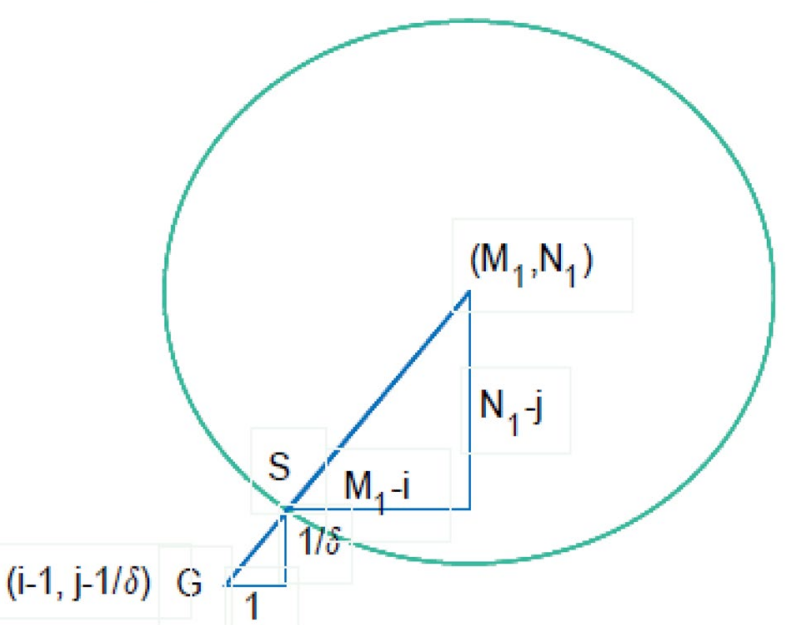

Fig. $15 S$ is a point on the circle adjacent to grid point $P$ and $G$ is a point where normal at $\mathrm{S}$ on cylinder cuts the grid 
numbers $(x, y)$ such that $x^{*}=(x-1) h, y^{*}=(y-1) h$. [we define grid number as (distance $x$ )/(grid length $h$ )]. We take centre of the circle $C$ at the grid numbers $\left(\frac{M+1}{2}, \frac{N+1}{2}\right)$. The corresponding coordinates for $C=\left(\frac{M-1}{2} h, \frac{N-1}{2} h\right)$. Let $r$ be the radius of the circle and $r=r_{0} h$. ( $r_{0}=$ number of intervals or grid numbers contained in $r$ ).

The equation of the circle $S:\left(x^{*}-\frac{M-1}{2} h\right)^{2}+\left(y^{*}-\frac{N-1}{2} h\right)^{2}=r^{2}$

$\Rightarrow\left(x-M_{1}\right)^{2}+\left(y-N_{1}\right)^{2}=r_{0}^{2}$ where $M_{1}=\frac{M+1}{2}, N_{1}=\frac{N+1}{2}$. Let the distance $P G=\left\{\begin{array}{c}a_{1} h, \text { if } G \text { is left to } P \\ b_{1} h, \text { if } G \text { is right to } P \\ c_{1} h \text {, if } G \text { is below } P \\ d_{1} h, \text { if } G \text { is above } P\end{array}\right.$ and $Q_{1}=\left\{\begin{array}{r}\left(x_{0}^{*},(j-1) h\right)-\text { coordinates } \\ \left(x_{0}, j\right)-\text { grid number }\end{array}\right.$ $Q_{2}=\left\{\begin{array}{r}\left((i-1) h, y_{0}^{*}\right)-\text { coordinates } \\ \left(i, y_{0}\right)-\text { grid number }\end{array}\right.$

Let $P_{1}(i, j+1), P_{2}(i, j-1), P_{3}(i+1, j)$ and $P_{4}(i-1, j)$ be the grid points adjacent to $P=(i, j)$ in the cavity. Grid numbers for the adjacent points of $\mathrm{P}$ are $\mathrm{PP}_{4}=a, \mathrm{PP}_{3}=b, \mathrm{PP}_{2}=c$ and $\mathrm{PP}_{1}=d$

\section{Calculating $\zeta$ on the cylinder when grid point $(i, j)$ is adjacent to cylinder:}

3rd Quarter of the circle If $C P_{3}<r_{0}$, then $x_{0}=M_{1}-\sqrt{r_{0}^{2}-\left(j-N_{1}\right)^{2}}$

$P Q_{1}=b=\left(x_{0}-i\right)=$ grid numbers and hence

$c_{1}=\frac{b\left(N_{1}-j\right)}{\sqrt{r_{0}^{2}-\left(j-N_{1}\right)^{2}}}$

$\therefore \psi_{G_{1}}=c_{1} \psi_{i, j-1}+\left(1-c_{1}\right) \psi_{i, j}$ by interpolating and $\zeta_{Q_{1}}=\frac{\psi_{G_{1}}}{h \sqrt{b^{2}+c_{1}^{2}}}$

If $C P_{1}<r_{0}$, then $y_{0}=N_{1}-\sqrt{r_{0}^{2}-\left(i-M_{1}\right)^{2}}$.

$P Q_{2}=d=\left(y_{0}-j\right)=$ grid numbers and hence

$a_{1}=\frac{d\left(M_{1}-i\right)}{\sqrt{r_{0}^{2}-\left(i-M_{1}\right)^{2}}}$

$\psi_{G_{2}}=a_{1} \psi_{i-1, j}+\left(1-a_{1}\right) \psi_{i, j}$ by interpolation and $\zeta_{Q_{2}}=\frac{\psi_{G_{2}}}{h \sqrt{d^{2}+a_{1}^{2}}}$

Similar derivations are used when a grid point $P$ is adjacent to the circle in 2 nd, 1 st and 4 th quarters of the circle.

\section{Calculating $\zeta$ on the cylinder when grid point $(i, j)$ is on the cylinder $\left(C P=r_{0}\right)$}

Let $\delta=\left|\frac{i-M_{1}}{j-N_{1}}\right|$
Where $P=S$ is the grid point on the cylinder If $\delta<1$, normal at $S$ cuts grid parallel to $x$-axis and If $\delta>1$, normal at $S$ cuts grid parallel to $y$-axis.

$$
D=\mathrm{GS}=\left\{\begin{array}{c}
h \sqrt{1+\delta^{2}}, \text { if } \delta<1 \\
h \sqrt{1+\frac{1}{\delta^{2}}}, \text { if } \delta>1
\end{array} \text { and } \zeta_{S}=\frac{\psi_{G}-\psi_{S}}{D}=\frac{\psi_{G}}{D},\right.
$$

since $\psi_{S}=0$ on the cylinder.

If $P=S$ is on the 3rd Quarter of the circle

if $\delta<1, \psi_{G}=\delta \psi_{i-1, j-1}+(1-\delta) \psi_{i, j-1}$ and $\zeta_{S}=\frac{\psi_{G}}{h \sqrt{1+\delta^{2}}}$

if $\delta>1, \psi_{G}=\frac{1}{\delta} \psi_{i-1, j-1}+\left(1-\frac{1}{\delta}\right) \psi_{i-1, j}$ and $\zeta_{S}=\frac{\psi_{G}}{h \sqrt{1+\frac{1}{\delta^{2}}}}$

Similar calculations follow for 2 nd, 1 st and 4 th quarters of the circle.

\section{References}

1. Williamson CHK (1989) Oblique and parallel modes of vortex shedding in the wake of a circular cylinder at low Reynolds numbers. J Fluid Mech 206:579-627

2. Henderson Ronald D (1995) Details of the drag curve near the onset of vortex shedding. Phys Fluids 7(9):2102-2104

3. Henderson Ronald D (1997) Nonlinear dynamics and pattern formation in turbulent wake transition. J Fluid Mech 352:65-112

4. Norberg C (2003) Fluctuating lift on a circular cylinder: review and new measurements. J Fluids Struct 17:57-96

5. Baranyi L, Lewis RI (2006) Comparison of a grid-based CFD method and vortex dynamics predictions of low Reynolds number cylinder flows. Aeronaut J 110(1103):63-71

6. Li Guoping, Humphrey Joseph A C (1995) Numerical modelling of confined flow past a cylinder of square cross-section at various orientations. Int J Numer Meth Fluids 20:1215-1236

7. Moukalled F, Acharya S (1996) Natural convection in the annulus between concentric horizontal circular and square cylinders. $J$ Thermo Phys Heat Transf 10(3):524-531

8. Cesini G, Paromcini M, Cortella G, Manzan M (1999) Natural convection from a horizontal cylinder in a rectangular cavity. Int J Heat Mass Transf 42:1801-1811

9. Breuer M, Bernsdorf J, Zeiser T, Durst F (2000) Accurate computations of the laminar flow past a square cylinder based on two different methods: lattice-Boltzmann and finite-volume. Int J Heat Fluid Flow 21:186-196

10. Salvatici E, Salvetti MV (2003) Large eddy simulations of the flow around a circular cylinder: effects of grid resolution and sub-grid scale modelling. Wind Struct 6(6):1-18

11. Projahn U, Rieger $\mathrm{H}$, Beer $\mathrm{H}$ (1981) Numerical analysis of laminar natural convection between concentric \& eccentric cylinders. Numer Heat Transf 4:131-146

12. Kim BS, Lee DS, Ha MY, Yoon HS (2008) A numerical study of natural convection in a square enclosure with a circular cylinder at different vertical locations. Int J Heat Mass Transf 51:1888-1906

13. Sahu Akhilesh K, Chhabra RP, Eswaran V (2009) Two dimensional unsteady laminar flow of a power law fluid across a square cylinder. J Nonnewton Fluid Mech 160:157-167 
14. Hussain SH, Hussein AK (2010) Numerical investigation of natural convection phenomena in a uniformly heated circular cylinder immersed in square enclosure filled with air at different vertical locations. Int Commun Heat Mass Transf 37:1115-1126

15. Berrone S, Garbero V, Marro M (2011) Numerical simulation of low Reynolds number flows past rectangular cylinders based on adaptive finite element and finite volume methods. Comput Fluids 40:92-112

16. Minguez M, Brun C, Pasquetti R, Serre E (2011) Experimental and high order LES analysis of the flow in near-wall region of a square cylinder. Int J Heat Fluid Flow 32:558-566

17. Lin Lu, Liu Ming-ming, Teng Bin, Cui Zhen-dong, Tang Guoqiang, Zhao Ming, Cheng Liang (2014) Numerical investigation of fluid flow past circular cylinder with multiple control rods at low Reynolds number. J Fluids Struct 48:235-259

18. Dey P, Das AK (2015) Numerical analysis of drag and lift reduction of square cylinder. Eng Sci Technol Int J 18:758-768

19. Ambreen T, Kim MH (2018) Flow and heat transfer characteristics over a square cylinder with corner modifications. Int J Heat Mass Transf 117:50-57

20. Ren Weiwei, Shu Chang, Yang Wenming (2013) An efficient immersed boundary method for thermal flow problems with heat flux boundary conditions. Int J Heat Mass Transf 64:694-705
21. Ashrafizadeh A, Hosseinjani AA (2017) A phenomenological study on the convection heat transfer around two enclosed rotating cylinders via an immersed boundary method. Int J Heat Mass Transf 107:667-685

22. Sarkar S, Ganguly S, Dalal A (2012) Analysis of entropy generation during mixed convective heat transfer of nano-fluids past a square cylinder in vertically upward flow. J Heat Transf 134:1225011-1225018

23. Yang Hu, Li Decai, Shu Shi, Niu Xiaodong (2017) Natural convection in a nano-fluid filled eccentric annulus with constant heat flux wall: a lattice Boltzmann study with immersed boundary method. Int Commun Heat Mass Transf 86:262-273

24. Williamson CHK (1996) Vortex dynamics in the cylinder wake. Annu Rev Fluid Mech 28:477-539

25. Biringen S, Chow CY (2011) An introduction to computational fluid mechanics by example. Wiley, Hoboken

26. Thom A (1933) The flow past circular cylinders at low speeds. Proc R Soc Lond A 141:651-669

Publisher's Note Springer Nature remains neutral with regard to jurisdictional claims in published maps and institutional affiliations. 